\title{
Antigenic and anticorpal diagnosis of invasive aspergillosis and candidosis in immunocompromised host
}

\author{
Marco Machetti, Anna Marchese
}

Sezione di microbiologia C.A. Romanzi, DISCAT - Università degli Studi di Genova, Genova, Italy

Key Words: aspergillosis, candidosis, diagnosis, antigens, antibodies

\section{Diagnosi antigenica ed anticorpale della aspergillosi invasiva e della candidosi nell'ospite compromesso}

\section{SUMMARY}

Invasive aspergillosis (IA) and candidemia are an increasing cause of morbidity and mortality in immunocompromised patients, but diagnostic procedures are often hampered by critical patients conditions. In recent years, non-culture methods have reached a standardization level suitable to be marketed and widely employed.

A method for a panfungal antigenic diagnosis, is the detection in serum of $(I \rightarrow 3)-B-D-G l u c a n$ (BDG), a polisaccaridic component of fungal cell wall. Best results can be obtained in Aspergillus, Candida and Fusarium infections, while the test performs poorly with Cryptococcus and in zygomycosis. The use of this method are limited by the high costs and by the need of disposable materials certified as glucan-free, in order to avoid false positive results.

Galactomannan (GM) antigen detection is a method for non-invasive diagnosis of IA. The assay, in latex agglutination (LA) and enzymeimmunoassay (EIA) format, detect GM in serum, whose presence correlates with IA. Sensitivity and specificity strongly fluctuate (50-100\% and $81-98 \%$ respectively) depending on the time of sampling, the positivity cut-off employed, the concomitant administration of antifungal drugs and of some antibiotics. Nevertheless the method is a very useful and widely employed tool for the diagnosis of IA.

For the diagnosis of candidemia, two Candida antigens may be detected in serum: the $56^{\circ} \mathrm{C}$ heat-labile antigen in LA format, and the mannan antigen, in LA and EIA format. Both the methods perform quite well, but sensitivities and specificities are not so good to allow a routinely and useful use in clinical practice.

Received June 26, 2007

Accepted October 8, 2007

\section{INTRODUZIONE}

Le infezioni micotiche vanno assumendo un ruolo sempre maggiore nelle malattie infettive, specialmente in soggetti malnutriti, sottoposti a prolungati ricoveri ospedalieri ed in generale immunocompromessi (62). L'attenuazione della capacità di esprimere un'efficiente risposta immunitaria è un forte fattore predisponente all'insorgenza di micosi opportunistiche, in cui organismi fungini normali commensali dell'organismo (es. Candida), se non innocui saprofiti dell'ambiente aereo (es. Aspergillus), divengono patogeni grazie ad un adattamento parassitario che in breve può portare a gravi complicanze, talvolta con esito letale (100).
Il calo nella risposta immunitaria può essere dovuto sia a fattori patologici, come emopatie maligne, sarcoidosi, linfomi, AIDS, sia a fattori esterni, quali l'esposizione a radioterapie locali, terapie antitubercolari, uso di antibatterici che favoriscono sovrainfezioni fungine, ma in particolare l'uso di farmaci immunosoppressivi nei pazienti sottoposti a trapianto (d'organo e di midollo osseo).

Ne consegue così un deficit dei granulociti neutrofili e un'alterazione dei meccanismi immunitari umorali e cellulari, tali da permettere il sopravvento degli organismi fungini (100).

Un ulteriore fattore di rischio, legato all'ospedalizzazione, è l'eventuale presenza di lavori edili in

\section{Corresponding author: Marco Machetti}


reparti limitrofi, che provocando il sollevamento e l'immissione nell'aria di grandi quantità di polveri, favorisce il rischio di micosi causate da funghi filamentosi quali Aspergillus, Fusarium e Mucor. Le micosi opportunistiche che si possono osservare con maggior frequenza sono quelle dovute a Candida e Aspergillus ed in misura minore a Cryptococcus.

La diagnosi classica di queste infezioni si basa sull'identificazione dei funghi in prelievi tissutali, eventualmente supportata dalla crescita in coltura, ma nei pazienti immunocompromessi le gravi condizioni cliniche spesso impediscono procedure diagnostiche invasive, e molte infezioni fungine sono così diagnosticate troppo tardi per un adeguato trattamento terapeutico, o addirittura al tavolo autoptico. Se l'autopsia non viene eseguita, molte infezioni non sono neppure identificate e pertanto è difficile anche valutarne la reale portata epidemiologica.

Per sopperire a questo deficit diagnostico da molti anni si studiano e si mettono a punto nuovi test diagnostici non invasivi, possibilmente rapidi, economici ed affidabili.

I target principali di questi nuovi sistemi sono gli antigeni ed i metaboliti fungini, le sequenze di acidi nucleici, mentre il rilevamento di anticorpi circolanti sembrerebbe poco appropriato viste le deboli risposte anticorpali che questi pazienti mostrano $(16,104)$.

Qui di seguito saranno descritti i principali metodi per la diagnosi antigenica non invasiva di candidemia e aspergillosi invasiva. In particolare saranno trattati quei test che sono stati validati e standardizzati ad un livello tale da essere disponibili in formato commerciale.

\section{Antigeni e metaboliti fungini}

Tutti i metodi che hanno avuto maggior diffusione e standardizzazione si basano sulla ricerca di antigeni e metaboliti fungini circolanti.

Attualmente sono disponibili sia sistemi panfungini, per una diagnosi generica di infezione micotica invasiva, sia metodi specie-specifici, utilizzabili laddove esiste il sospetto diagnostico di una infezione fungina specifica.

\section{Diagnosi antigenica panfungina:} ricerca dell' $(\mathbf{1} \rightarrow \mathbf{3})$ - $\mathbf{\beta}$-D-Glucano

L' $(1 \rightarrow 3)-\beta$-D-Glucano (BDG) è un polisaccaride, componente specifico della parete cellulare fungi- na, a totale eccezione degli zigomiceti, e parziale del Cryptococcus, che lo esprime in forma ridotta. Il test per il rilevamento del BDG circolante è basato sulla capacità del glucano di attivare il fattore $\mathrm{G}$, componente della cascata coagulatoria del lisato amebocitario del granchio a ferro di cavallo, Limulus polyphemus e Tachypleus tridentatus (principio simile a quello dei test per il rilevamento dell'endotossina - LAL test).

Il metodo, realizzato in formato cromogenico su micropiastra è stato messo a punto ed introdotto sul mercato giapponese a metà degli anni ' 90 ("FungiTec G test" - Seikagaku Corporation, Tokyo, Japan), mostrando nello studio pilota nipponico buone sensibilità $(90 \%)$ e specificità $(100 \%)$ (68). Ciò nonostante, a causa dei costi elevati del test (30-40 €/campione) e di una procedura tecnica ad alto rischio di inquinamento e alterazione dei risultati, il test non ha quasi mai varcato i confini giapponesi, e per molti anni quasi tutti gli studi pubblicati vedevano coinvolti i produttori stessi del metodo.

Più recentemente, da parte della consociata statunitense del produttore giapponese (Associates of Cape Cod, E. Falmouth, MA, U.S.A.), sono state

Figura I. Principio alla base del test per la ricerca del $(I \rightarrow 3)-\beta$-D-Glucano. Cascata coagulatoria del lisato amebocitario del granchio a ferro di cavallo, Limulus polyphemus e Tachypleus tridentatus (da Fungitell test/Associates of Cape Cod, E. Falmouth, MA, U.S.A. - insert sheet)

prodotte due nuove versioni del test ("Glucatell", per utilizzo a scopo di ricerca e "Fungitell", per utilizzo diagnostico). Una leggera semplificazione tecnica e la concomitante approvazione del test da parte della Food and Drug Administration statunitense hanno favorito la diffusione del test sia negli U.S.A. sia in Europa. In Figura I è mostrato il principio del test così come riportato nelle istruzioni del kit "Fungitell".

Nonostante la semplificazione della procedura del test, un certo rischio di contaminazione ed alterazione dei risultati sussiste ancora, anche se un training adeguato del personale addetto all'esecuzione del test e l'adozione di alcuni particolari accorgimenti permettono di ottenere risultati affidabili e ripetibili. Esiste in ogni caso un problema intrinseco al 
sistema, già evidenziato nelle prime versioni giapponesi, ossia la necessità di usare materiale speciale da laboratorio, libero da glucano, poiché questa molecola si ritrova normalmente adesa alla plastica dei più comuni strumenti di laboratorio (pipette monouso, puntali, provette, micropiastre, ecc). Questo richiede quindi l'utilizzo di materiale certificato "glucano free" che aumenta ulteriormente i costi del test.

Il test "Fungitell" è da considerare positivo quanto il BDG rilevato è $>80 \mathrm{pg} / \mathrm{ml}$, indeterminato quando compreso tra 60 e $80 \mathrm{pg} / \mathrm{ml}$, negativo quando $<60 \mathrm{pg} / \mathrm{ml}$. Alcuni autori però utilizzano il livello di $60 \mathrm{pg} / \mathrm{ml}$ quale cut-off di positività, a leggero discapito della specificità del sistema.

Un recente studio multicentrico effettuato su 163 pazienti con infezione fungina invasiva e 170 pazienti di controllo, ha confermato una buona sensibilità per Aspergillus, Candida e Fusarium (rispettivamente $80 \%, 81.3 \%$ e $100 \%$ ) e l'incapacità totale $\mathrm{o}$ fortemente ridotta di rilevare infezioni da zigomiceti (0\%) e Cryptococcus (25\%) (70). Nello stesso studio inoltre, non sono stati dimostrati eventuali effetti della terapia antifungina sulla sensibilità del test per il BDG $(p=0.69)$.

La ricerca del BDG non è comunque indenne da possibili interazioni. In uno studio effettuato su 46 pazienti di terapia intensiva è stato riportato che batteriemie documentate possono produrre falsi positivi per il BDG (17). Analogo risultato viene da uno studio del 2005 effettuato su 156 pazienti, tra i quali, 14 pazienti su 25 con batteriemie documentate (10/14 per cocchi Gram positivi) erano falsamente positivi per il BDG pur in assenza di infezione fungina (73).

Anche alcuni filtri e membrane di cellulosa utilizzati per l'emodialisi sembrano in grado di dare test fortemente positivi per il BDG (70).

In merito a falsi positivi per BDG dovuti a trattamenti con farmaci $\beta$-lattamici, fattore che come vedremo in seguito è di notevole rilievo nella diagnostica specie specifica di aspergillosi, sono riportati sia dati a favore di una cross-reazione con l'Amoxicillina/Clavulanato (59) sia dati che indicano un'assenza di interazioni tra farmaci e test (73). Un $20 \%$ di falsi negativi dovuti a valori anomali di emoglobina e sempre un $20 \%$ di falsi positivi dovuti a valori anomali di bilirubina e di trigliceridi sono stati descritti recentemente (73).

Un'ulteriore versione della ricerca del BDG è il "Wako-test" (Wako Pure Chemical Industries, Ltd., Tokyo, Japan), test cinetico sempre di origine giapponese. In uno studio nipponico questo test cinetico è apparso meno sensibile $(72.7 \%$ vs. $87.9 \%$ ) ma più specifico rispetto a quello cromogenico (108). In un altro studio giapponese effettuato su pazienti ematologici a rischio di aspergil- losi invasiva, il "Wako-test" ha fornito scarsi valori di sensibilità $(55 \%)$ ma buona specificità $(98 \%)$ e la tendenza a positivizzare tardivamente durante il decorso clinico di AI (41).

In conclusione le informazioni disponibili sul test per il BDG fanno ritenere che questo metodo abbia buone potenzialità, ma molti aspetti del test, in particolare il suo impatto clinico, devono ancora essere valutati. Ulteriore punto da studiare è anche l'eventuale integrazione del test per il BDG con metodi antigenici/anticorpali specie specifici.

\section{Diagnosi specie-specifica di infezioni da Candida}

Per la diagnosi di candidosi disseminata sono stati studiati molti antigeni, metaboliti ed anticorpi, quali possibili marker di infezione da Candida.

In merito alla ricerche di anticorpi, in genere queste sono considerate poco utili per la diagnosi delle micosi opportunistiche invasive, poiché i pazienti immunocompromessi non sembrano in grado di mostrare risposte anticorpali degne di rilievo. In alcuni studi risalenti agli anni 80 e 90 , vari metodi per il dosaggio di anticorpi antiCandida sono risultati poco sensibili (25-29\%) in pazienti immunocompromessi $(36,71)$. Altri studi hanno dato risultati più incoraggianti. Il dosaggio di anticorpi per una metalloproteina da $52 \mathrm{kDa}$ di Candida albicans ha fornito sensibilità e specificità pari al 83\% e 97\% seppur in pazienti dei quali lo studio non specifica lo stato di immunitario.

Un altro lavoro ha studiato la ricerca di anticorpi verso l'aspartil proteinasi di Candida albicans, un fattore di virulenza rilasciato dal micete durante l'invasione tessutale. In una valutazione di 33 pazienti con candidosi invasiva le sensibilità e specificità del test sono state rispettivamente del $70 \%$ e $76 \%$ (67). Nessuno di questi metodi ha però finora trovato un'evoluzione e standardizzazione di tipo commerciale.

In accordo con questi ultimi studi, alcune ricerche recenti hanno comunque mostrato che anche nei pazienti immunocompromessi può essere presente una risposta anticorpale, il cui dosaggio, effettuato in abbinamento con quello di frazioni antigeniche di Candida, migliora le performance globali della diagnostica non invasiva per infezioni da Candida (20). Quest'ultimo utilizzo delle ricerche anticorpali, sarà approfondito nelle specifiche sezioni relative alle ricerche antigeniche di Candida.

\section{Rilevamento di antigeni di Mannano per la diagnosi di candidosi invasiva}

Da molti anni si studia il rilevamento del mannano (MAN), antigene polisaccaridico della parete cellulare di Candida, nel siero di pazienti affetti da candidosi disseminata. Le tecniche utilizzate sono 
diverse: radioimmunologiche (Radio Immuno Assay - RIA), immunoenzimatiche (EnzimeLinked Immunosorbent Assay - ELISA) ed in lattice agglutinazione (LA) $(77,104)$.

Qualunque sia il metodo utilizzato, è necessario un pre-trattamento del campione per dissociare $\mathrm{i}$ complessi circolanti antigene-anticorpo, che sottrarrebbero una cospicua frazione antigenica all'analisi. Questo si realizza mediante trattamenti idrolitici, enzimatici, con EDTA e bollitura, i quali peraltro non alterano il MAN, essendo l'antigene pronasi resistente e termostabile $(16,77)$.

All'inizio degli anni '90 è stato messo in commercio un sistema in LA, "Pastorex Candida" (Sanofi Diagnostics Pasteur, Marnes-La-Coquette, France) per il rilevamento di un residuo $\alpha$-linked oligomannosidico del MAN ( $\alpha$-MAN) su siero. A causa del formato stesso del test e della breve permanenza in circolo del MAN, la sensibilità del metodo è variabile e tendenzialmente bassa (30$50 \%$ ) e pertanto questo sistema non gode di una grande diffusione $(30,64,74)$.

Sul finire degli anni ' 90 , a partire dallo stesso anticorpo monoclonale (EBCA1) impiegato nel test "Pastorex Candida", la Bio-Rad (Ex Sanofi Diagnostic Pasteur, Marnes-La-Coquette, France) ha prodotto e commercializzato sia una versione ELISA del test per la ricerca dello stesso antigene MAN ("Platelia Candida Ag"), sia un test ELISA per la ricerca di anticorpi anti-MAN ("Platelia Candida AB"). Nel "Platelia Candida Ag" il risultato è quantitativo ed espresso in $\mathrm{ng} / \mathrm{ml}$ di MAN circolante. Il test è negativo se il risultato è $<0.25$ $\mathrm{ng} / \mathrm{ml}$, intermedio se $\geq 0.25$ e $<0.5$, positivo quando $\geq 0.5$. Nel "Platelia Candida AB" il risultato è quantitativo ma espresso in unità arbitrarie (AU) definite dal produttore. Il test è negativo se il risultato è $<5 \mathrm{AU}$, intermedio $\mathrm{se} \geq 5 \mathrm{e}<10 \mathrm{AU}$, positivo quando $\geq 10 \mathrm{AU}$.

Il primo studio (francese) che ha valutato l'impiego contemporaneo di questi due metodi ha riacceso l'attenzione sulla ricerca di anticorpi antifungini, che generalmente era considerata poco utile nei tutti immunodepressi. Lo studio, effettuato su 43 pazienti con candidosi documentate, ma non strettamente immunocompromessi bensì con svariate malattie di base (patologie gastrointestinali, cardiomiopatie, tumori solidi, trapianti d'organo, malattie ematologiche), ha mostrato che mentre le ricerche del MAN o di anticorpi anti-MAN considerate singolarmente avevano sensibilità piuttosto deludenti (40-50\%), la sensibilità totale, ottenuta accorpando i risultati di entrambi i sistemi, saliva al $80 \%$, con una specificità del $93 \%(84)$.

In seguito gli stessi autori hanno evidenziato che la sensibilità totale dei due test usati contemporaneamente varia a seconda della specie di Candida coinvolta (C. albicans 100\%, C. glabrata 83\%, C. tropicalis $80 \%$, C. krusei e C. kefyr 50\%,C. parapsilosis $40 \%$ ) (83).

Per sopperire alla scarsa sensibilità del test "Platelia Candida Ag" per l' $\alpha$-MAN, dovuta alla clearance rapida dalla circolazione di questo tipo di MAN, nel 2004 gli stessi ricercatori francesi hanno realizzato un nuovo test ELISA per la ricerca di un residuo $\beta$-linked oligomannosidico del MAN ( $\beta$-MAN) su siero. L'ipotesi era che i due tipi di MAN potessero essere espressi in maniera diversa a seconda della specie e ceppo di Candida, interagissero differentemente con il sistema immunitario ed avessero anche una clearance diversa. Tali ipotesi sembrerebbero confermate da uno studio retrospettivo effettuato su 26 pazienti (90 campioni di siero), il quale ha evidenziato che i test per l' $\alpha$-MAN e per il $\beta$-MAN, considerati singolarmente, avevano una scarsa sensibilità $(69 \%$ entrambi) ed alta specificità (rispettivamente $98 \%$ e 95\%), mentre la sensibilità complessiva dei due test migliorava notevolmente $(85 \%)$ e la specificità restava al 95\% (82).

Il test "Platelia Candida Ag" è stato anche utilizzato sul liquor di 5 pazienti con sospetta meningite da Candida. In 4 di questi, tutti con candidosi documentata, il test è risultato positivo, mentre nel quinto (candidosi sospetta) e nei 3 pazienti di controllo, il test è risultato negativo. Vista l'esigua casistica dello studio i risultati ottenuti hanno un significato clinico limitato ma sono sicuramente incoraggianti per questo tipo di patologia (97).

Nel 2005 è stato anche riportato che in pazienti con lesioni epatospleniche, l'uso combinato dei test "Platelia Candida" Ag e AB può abbreviare il tempo medio della diagnosi di candidosi rispetto alla diagnosi radiologica ( 9 vs. 25 giorni dopo l'insorgenza di febbre quale primo segno clinico di infezione; $p<0.001)(75)$.

L'uso di questi test non è ovviamente immune da problematiche.

Una di queste, finora evidenziata solo in vitro, è la reazione crociata del test per l'antigene MAN con due microrganismi fungini, Geotrichum candidum e Fusarium verticillioides (78).

Di maggior peso è quanto riportato in uno studio scozzese del 2005 effettuato su un gruppo di pazienti ematooncologici. Il test "Platelia Candida $\mathrm{Ag}$ " è risultato infatti pressoché inutilizzabile poiché il $61 \%$ dei kit impiegati aveva fallito i controlli interni di validazione, inoltre circa il $30 \%$ dei risultati rimanenti non era riproducibile (3).

I lavori esistenti sui kit commerciali per l'antigene MAN ed anticorpi anti-MAN non sono finora molto numerosi, ma alcuni di questi danno suggerimenti circa l'uso corretto di tali metodi. E' stato infatti riportato come in pazienti ematologici con 
candidemia documentata, la positività del MAN possa anticipare mediamente di una settimana la positivizzazione delle emocolture. Alla positività delle emocolture però, l'antigene MAN potrebbe non essere più rilevabile mentre si potrebbe rilevare un incremento degli anticorpi anti-MAN (39, 84). Al contrario, in pazienti chirurgici non neutropenici è la risposta anticorpale che può precedere la positività delle emocolture nel $73 \%$ dei pazienti. Alla positivizzazione delle emocolture gli anticorpi anti-MAN decadono e si può assistere all'incremento dell'antigene MAN (107). Tutto questo suggerisce che i test per il MAN ed antiMAN potrebbero essere poco utili se effettuati solo al momento della positività di un'emocoltura per Candida, ma dovrebbero essere usati come strumenti di monitoraggio preventivo, seriato e continuativo, sui pazienti a rischio per candidosi. Resta ancora da chiarire la possibile influenza di una colonizzazione da Candida sul test per il MAN, ed in particolare su quello per gli anticorpi anti-MAN. Una recente segnalazione presentata al $16^{\circ}$ congresso ECCMID, riguardante l'impiego di questi due metodi su 46 pazienti sottoposti a trapianto di fegato, indica che la colonizzazione da Candida sembra influenzare attivamente la positività dei test per il MAN ed anti-MAN. Infatti 26/46 pazienti erano colonizzati da Candida, e di questi 26 , ben $14(53.8 \%)$ erano positivi per il MAN e/o anti-MAN, rispetto a 6 positivi su 20 $(30 \%)$ tra i pazienti non colonizzati. Benché la differenza non sia statisticamente significativa (test $\chi^{2} ; p=0.139$ ), i dati suggeriscono che la probabilità di avere un test positivo per MAN e/o anti-MAN nei pazienti colonizzati da Candida sia più che doppia rispetto a quella dei pazienti non colonizzati (Odds Ratio $=2.72$; 95\%; Interv. Conf. 0.797-9.296) (45).

\section{Rilevamento di antigeni termolabili a $56^{\circ} \mathrm{C}$ per la diagnosi di candidosi invasiva}

In aggiunta ai test basati sull'antigene MAN, esiste un altro metodo commerciale volto a rilevare un antigene di Candida non caratterizzato a livello molecolare, le cui caratteristiche (sensibilità al calore e al trattamento con pronasi, incapacità di reagire con sistemi per il rilevamento del MAN) permettono di escludere che si tratti dello stesso MAN e indirizzano verso una natura glicoproteica. Si ipotizza che questo sistema rilevi o un neo-antigene derivato dal processamento della Candida da parte dell'ospite, oppure un antigene non specifico dell'ospite che cross-reagisce con il lievito (24).

La forma commerciale del test è una LA di semplice esecuzione, il "Cand-Tec test" (Ramco Laboratories, Houston, Tex.), ma la sua utilità è controversa avendo fornito risultati alterati da falsi positivi e basse sensibilità anche quando comparato con la ricerca del MAN in LA $(16,30,104)$. Lavori più recenti ne hanno confermato la sensibilità piuttosto bassa e molto variabile $(37-77 \%)$ $(2,64,69)$ ed uno studio italiano del 1999, effettuato su 214 pazienti di terapia intensiva, ha riportato un bassissimo valore predittivo positivo (13$17 \%$ ) dei risultati del test "Cand-Tec" (71).

In controtendenza a tali riscontri negativi c'è uno studio recente effettuato durante 11 episodi febbrili correlati a chemioterapia post-remissione in 10 pazienti con leucemia acuta. Il test "Cand-Tec" è stato impiegato seriatamente sia prima che dopo l'inizio della chemioterapia, e l'aumento dell'antigenemia per Candida, unitamente all'insorgenza di neutropenia febbrile, è stato utilizzato come indicazione per l'inizio di un trattamento antifungino. Nei 9 episodi in cui la terapia antifungina ha avuto esito positivo, il test "Cand-Tec" ha mostrato un calo dell'antigenemia fino ai livelli pre-chemioterapia. Nei 2 episodi in cui la terapia antifungina è risultata inefficace, i livelli dell'antigene non sono calati. È stato quindi suggerito che il test "Cand-Tec" possa essere un valido supporto per prevenire un impiego eccessivo di farmaci antifungini (35).

Probabilmente sulla scia degli analoghi lavori su MAN ed anti-MAN, in uno studio del 2002 effettuato su 104 pazienti in terapia intensiva, il "Cand-Tec" è stato usato congiuntamente con un test in emoagglutazione indiretta per il rilevamento di anticorpi anti-Candida (Labor Diagnostica, Heiden, Germany). In 17 pazienti con sospetta infezione da Candida, la sensibilità e specificità dell' antigenemia erano del $58.8 \%$ e $97.6 \%$, mentre per gli anticorpi anti-Candida erano pari al $52.9 \%$ ed al $85.7 \%$. Le sensibilità e specificità totali dei due test erano invece pari al $100 \%$ ed al $83,3 \%$, con un incremento ottimale della sensibilità seppure a lieve discapito della specificità (6). Nel 2003 è stato anche descritta una nuova versione colorimetrica del test "Cand-Tec", il "CandTec MT“ (Ramco, Japan), in cui l'agglutinazione al lattice avviene in micropiastra, e le variazioni dell'assorbanza dei campioni a $490 \mathrm{~nm}$ sono lette fotometricamente. Il "Cand-Tec MT", usato su 25 pazienti ematologici, ha avuto una sensibilità del $100 \%$ nel rilevare i casi di infezione profonda da Candida, mentre la specificità si è attestata sull' $80 \%$ (63). Inoltre i risultati del nuovo test sembrano ben correlare con gli effetti delle terapie antifungine. Purtroppo, questo resta l'unico lavoro in cui tale test è stato studiato.

\section{Diagnosi specie-specifica di infezioni da Aspergillus}

La ricerca di antigeni circolanti per la diagnosi di aspergillosi invasiva (AI) è un metodo impiegato 
da molti anni, la cui correlazione con l'infezione da Aspergillus è ben documentata, anche se esistono alcune difficoltà dovute sia all'ubiquità di questi funghi sia alla sensibilità e specificità dei sistemi diagnostici (16).

Sono stati studiati numerosi metodi (ELISA, RIA, LA) per rilevare antigeni di Aspergillus in campioni biologici diversi di pazienti con AI (siero, urine, lavaggi bronco alveolari, liquor) ed i risultati mostrano sensibilità piuttosto variabili (3095\%) $(16,21,43,89,106)$. Spesso le tecniche hanno soglie di rilevamento troppo alte e gli antigeni possono essere rilevati per brevi periodi, per la formazione di immunocomplessi rapidamente eliminati via endocitosi dalle cellule del Kupffer epatiche (16). Come nel caso della ricerca del MAN per Candida, una parziale soluzione viene dai trattamenti applicabili al siero per dissociare gli immunocomplessi mediante diluizioni, riscaldamento, impiego di sostanze acide.

\section{Rilevamento di antigeni di galattomannano per la diagnosi di aspergillosi invasiva}

Fra tutti le componenti cellulari di Aspergillus, il galattomannano (GM), frazione polisaccaridica della parete cellulare, è quella più studiata in quanto la sua presenza nel siero correla con stati di AI in atto $(11,76)$. Da molti anni quindi il test per il GM è un utile supporto alla diagnosi di AI $(18,79)$.

Come per il MAN per Candida, per la diagnosi di AI sono commercializzati due metodi basati sullo stesso anticorpo monoclonale (EB-A2), uno in LA, "Pastorex Aspergillus" (BioRad, Marnes-LaCoquette, France) ed uno in ELISA, "Platelia Aspergillus" (BioRad, Marnes-La-Coquette, France), che è un'evoluzione della versione in LA, e ne ha abbassato la soglia di rilevamento del $\mathrm{GM}$ da $15 \mathrm{ng} / \mathrm{ml}$ ad $1 \mathrm{ng} / \mathrm{ml}$.

Nei primi anni '90, in cui era disponibile solo il test in LA, il "Pastorex Aspergillus" ha trovato subito una larga diffusione e si è dimostrato molto specifico $(90-100 \%)$ ma con una sensibilità alquanto variabile e tendenzialmente bassa (36$95 \%)(32,43,106)$. Dal 1997 è stato commercializzato il test ELISA "Platelia Aspergillus", che fin dai primi studi di confronto con il metodo in LA si è dimostrato, come prevedibile, più sensibile del suo predecessore $(46,90,99)$. Attualmente il metodo ELISA è quello più utilizzato per la ricerca del GM e dopo molti anni di impiego pressoché esclusivo in Europa ed Asia, nel 2003 il test è stato approvato dalla Food and Drug Administration statunitense ed introdotto anche negli U.S.A..

La ricerca del GM è impiegata principalmente nei pazienti ematooncologici $(12,13,26,51)$, per i quali ne è stata studiata l'applicazione come crite- rio diagnostico e fattore decisionale per guidare l'approccio terapeutico (unitamente a valutazioni cliniche e strumentali) $(52,85)$.

Generalmente se ne raccomanda un uso seriato e continuativo, con una frequenza di raccolta di $2 / 3$ campioni la settimana, per aumentare la probabilità di rilevare il GM circolante $(43,57)$.

Nonostante l'evoluzione tecnica dal sistema LA a quello ELISA, anche la sensibilità di quest'ultimo è molto variabile $(29-100 \%)$ e la specificità (81$98 \%$ ) risulta affetta dalla possibilità di avere falsi positivi causati da molteplici fattori (31).

Ad influenzare i risultati dell'ELISA per GM concorrono moltissimi elementi, fondamentalmente raggruppabili in due categorie: biologici ed epidemiologici. Tra i primi ci sono il sito di infezione, la specie di Aspergillus coinvolta, la profilassi e/o terapia antifungina, le condizioni di base ed il grado di immunosoppressione, la funzionalità epatica e renale, la presenza in circolo di anticorpi anti-Aspergillus, le modalità di raccolta e conservazione dei campioni di siero da testare, le procedure seguite nel test. I principali fattori epidemiologici sono invece la popolazione dei pazienti, le strategie di raccolta dei campioni da analizzare, le definizioni di "paziente con infezione" e di "risultato positivo", la prevalenza dell'infezione, i cutoff utilizzati per la positività del campione, e l'esperienza del laboratorio che effettua il test (57). È evidente che la situazione è davvero complessa e le diverse combinazioni di tutte queste variabili possono influire sulle performance diagnostiche del test, spiegando la fortissima variabilità che emerge dalla mole notevole di studi che a tutt'oggi sono stati effettuati sul "Platelia Aspergillus".

Il test è stato anche impiegato su pazienti sottoposti a trapianto d'organo (fegato, rene e polmone) con risultati meno incoraggianti di quelli ottenuti su pazienti ematologici $(22,34,42)$ anche se recentemente è stato suggerito che la ricerca del GM può essere un utile strumento di guida della “preemptive therapy" per la prevenzione dell'AI nei pazienti trapiantati d'organo (53).

Oltre che sul siero la ricerca del GM è stata anche usata su lavaggio broncoalveolare, con risultati interessanti $(8,81,86)$, sulle urine con performance meno promettenti $(81,33)$ e su liquor, particolare applicazione grazie alla quale il test sembra essere specifico per l'aspergillosi cerebrale $(38,98,103)$. In merito alla validità della ricerca del GM in pediatria sono stati prodotti pochi lavori, la maggior parte dei quali con casistiche piuttosto esigue. L'indicazione generale è abbastanza buona, ed il metodo appare molto sensibile $(100 \%)$ ma con qualche problema di specificità $(50-90 \%)(10,44$, $47,80,90,92)$. In genere tale aspecificità sembra dovuta a cause e fattori strettamente pediatriche, 
come sarà discusso in seguito.

Un fattore importante, tuttora oggetto di valutazioni e continui aggiornamenti, è il cut-off di positività del test ELISA, che nel corso degli anni è passato dall'originario indice GM 1.5 (indice GM = rapporto tra la densità ottica a 450/620 nm del campione testato e quella del controllo cut-off fornito nel test; $1 \mathrm{ng} / \mathrm{ml} \mathrm{GM}$ ), all'attuale $0.7-0.5$, valori che incrementano la sensibilità del test senza avere eccessivi scadimenti della specificità $(29,50,54)$. A tutt'oggi sono state descritte diverse cause di risultati falsi negativi (scarsa sensibilità) e falsi positivi (scarsa specificità) del test per il GM, qui riportate in Tabella 1.

Tra $i$ fattori in grado di abbassare la sensibilità del test per il GM, due sono di particolare rilevanza. Uno consiste nella concomitante presenza, inaspettata in pazienti immunocompromessi, di anticorpi anti-Aspergillus. Uno studio del 2002 ha infatti mostrato che la presenza di anticorpi antiAspergillus nel 36\% dei pazienti immunocompromessi esaminati (54/150 episodi di AI, possibile, probabile e documentata) ha coinciso con una sensibilità inferiore del test per il GM rispetto a pazienti privi di risposta anticorpale $(p=0.04)$.

E probabile che la presenza di anticorpi antiAspergillus possa sottrarre una considerevole quantità di antigene GM al rilevamento con il test ELISA e ciò suggerirebbe la necessità di abbinare al test per il GM una ricerca di anticorpi antiAspergillus, al fine di interpretare meglio eventuali falsi negativi per il GM (29).

L'altra importante causa di falsi negativi è l'uso di profilassi o terapia antifungina durante il monitoraggio per il GM. Nel corso degli anni di impiego del test per il GM sono state molte le segnalazioni aneddotiche in tal senso, ma solo recentemente uno studio ha affrontato direttamente il problema. Quarantasei pazienti con IA (272 sieri) e 269 pazienti di controllo (3005 sieri) sono stati valutati con il "Platelia Aspergillus" alla luce di un'eventuale somministrazione (profilassi/terapia) di farmaci antifungini (Itraconazolo, Amfotericina B desossicolato e liposomiale, Voriconazolo, Caspofungina). Non è stata effettuata una valutazione differenziata per singolo farmaco ma solo complessiva. Il dato che emerge è comunque molto chiaro poiché nella prima settimana dal giorno della diagnosi di AI, la sensibilità del test (cut-off indice GM 0.5) tra i pazienti in profilassi/terapia antifungina era del $52 \%$ contro una sensibilità dell' $89 \%$ per i pazienti che non ricevevano antifungini $(p=0.02)$. Questo dato è di estrema importanza ed implica che il monitoraggio per l'AI con il test per il GM sia valutato alla luce di concomitanti terapie antifungine (55).

In merito ai falsi positivi, le cause descritte sono moltissime, ed alcune sembrano essere pressoché esclusive dei pazienti pediatrici (GM nei cibi, in formulazioni particolari di latte in polvere, per colonizzazione intestinale da Bifidobacterium spp.) come riportato in Tabella 1.

Inoltre svariati microrganismi possono dare reazioni positive per il GM, di cui alcune verificate solo in vitro (Acremonium spp., Alternaria alternata, Alternaria spp., Botrytis tulipae,

Tabella I. Principali cause di false negatività e false positività del test per l'antigene GM di Aspergillus

\begin{tabular}{lc}
\hline Falsi negativi nella ricerca dell'antigene GM & Rif. \\
\hline Carico fungino ridotto, potenziale angioinvasivo modesto & 9,57 \\
\hline Rilascio limitato di GM (alterazioni dei nutrienti e pH dell'ospite) & 43,57 \\
\hline Elevato titolo anticorpale anti-Aspergillus & 29 \\
\hline Concomitante profilassi o terapia antifungina & $8,55,66$ \\
\hline
\end{tabular}

Falsi positivi nella ricerca dell'antigene $\mathbf{G M}$

Rif.

\begin{tabular}{lc}
\hline Reazioni crociate con altri microrganismi & $14,25,40,9,, 95$ \\
\hline Trattamenti con ciclofosfamide (su urine in modello animale di Al) & 28 \\
\hline GVHD cronica (causa probabile: paraproteine o anticorpi auto-reattivi) & 27 \\
\hline Antigenemia transitoria senza Al, in particolare durante neutropenia & 37 \\
\hline Cotone di tamponi per prelievi bioptici & 15 \\
\hline Pazienti con insufficienza renale cronica emodializzati & 19 \\
\hline Neonati prematuri & 87 \\
\hline Neonati prematuri per acido lipoteicoico di Bifidobacterium spp. & 58 \\
\hline Neonati prematuri per latte in polvere di origine bovina & 23 \\
\hline GM nei cibi (farine, riso, pasta, soia), per danno mucosa intestinale, & $4,44,65$ \\
\hline in particolare in pazienti pediatrici & $\mathrm{I}, 4,5,7,48,49,56$, \\
\hline GM in farmaci B-lattamici, (rilevabile nel siero dei pazienti trattati & $60,61,72,88,94$, \\
e nei preparati antibiotici, particolarmente Piperacillina/Tazobactam, & $96,102,105$ \\
Amoxicillina/Clavulanato, Amoxicillina, Ampicillina) &
\end{tabular}


Cladosporium cladosporoides, Cladosporium herbarum, Cryptococcus neoformans, Fusarium oxysporum, Geotrichum capitatum, Paecilomices variotii, Penicillium chrysogenum, Rhodotorula rubra, Trichophyton interdigitalis, Trichophyton rubrum, Wallemia sebi, Wangiella dermatitidis) $(14,25,40,91,95)$.

Dal 2003 però, una particolare fonte di test GM falsi positivi ha suscitato grande interesse, ed in questi pochi anni molte ricerche hanno ben documentato il problema. In realtà il fenomeno fu segnalato già nel 1997 quando si verificò che il test "Pastorex Aspergillus" risultava positivo su alcuni farmaci in vitro (Piperacillina $\mathrm{e}$ Amoxicillina/Clavulanato) (4).

Tale osservazione non ebbe alcun seguito, né laboratoristico né clinico fino al 2003, quando, al $43^{\circ} \mathrm{C}$ congresso ICAAC due abstract hanno riportato osservazioni cliniche inerenti a falsi positivi per GM con il test ELISA in pazienti trattati con Piperacillina/Tazobactam $(93,101)$. In seguito moltissimi lavori hanno delineato e chiarito il quadro della situazione.

Sostanzialmente è stato documentato che il trattamento con farmaci $\beta$-lattamici, in particolare $\mathrm{P}$ i p e r a c i $11 \mathrm{i} \mathrm{na/T} \mathrm{a} \mathrm{z}$ o b a c t a m, Amoxicillina/Clavulanato, Amoxicillina, Ampicillina, può produrre test molto positivi per il GM anche in pazienti privi di alcun segno di AI $(1,56,61,88,94,102)$. La positività è riscontrabile sugli stessi farmaci saggiati in vitro $(75-80 \%$ dei lotti di Piperacillina/Tazobactam) $(1,5,7,48$, $56,88,94,102)$. La cinetica del fenomeno indica che trattamenti prolungati con $\beta$-lattamici (oltre 23 somministrazioni) producono innalzamenti del test per il GM immediatamente dopo la somministrazione, con una tendenza all'accumulo del segnale dopo ripetute somministrazioni. Dopo trattamenti prolungati, il GM può rimanere positivo anche per 5-7 giorni dopo l'interruzione del trattamento antibatterico $(5,7,49,88,105)$. L'origine molecolare di questi falsi positivi è quasi certamente da attribuire alla presenza nei farmaci antibatterici di residui antigenici simili al GM (o vero GM) derivanti dai processi fermentativi impiegati per produrre gli antibiotici (60).

Questa interazione comporta particolari problemi nella gestione di pazienti a rischio di AI che necessitino anche di trattamenti antibiotici con $\beta$-lattamici. In tali casi infatti i clinici potrebbero dover scegliere tra variare il trattamento antibiotico per non alterare la veridicità del test per il GM, oppure somministrare comunque gli antibiotici più idonei al caso rischiando una risposta inaffidabile dell'antigenemia per GM.

In conclusione, la ricerca del GM è un test di facile esecuzione ma di difficile interpretazione. Per un uso corretto ed una valutazione attendibile dei risultati del GM, è ormai imprescindibile la conoscenza di tutte le variabili che possono influire sul test. Ciò nonostante la ricerca dell'antigene GM rappresenta un notevole passo avanti per la diagnosi dell'AI nel paziente immunocompromesso. A fronte della mancanza di crescita di Aspergillus in emocoltura e della difficoltà di ottenere una diagnosi istologica di AI, la positività del test ELISA per GM contribuisce giornalmente ad effettuare diagnosi di aspergillosi invasiva in molti ospedali, contribuendo a migliorare le probabilità di sopravvivenza e la qualità di vita dei pazienti a rischio per questa temibile patologia.

\section{Integrazione dei test panfungini e specie-specifici}

Come visto finora, attualmente è possibile fare diagnosi antigenica/anticorpale di micosi invasiva opportunistica sia con metodi specie-specifici che con metodi panfungini. In un approccio diagnostico razionale, un test panfungino dovrebbe essere utilizzato quale test di screening per infezione micotica, e ad una sua positività dovrebbe seguire un'indagine specie-specifica, indirizzata verso una specifica eziologia fungina sulla base del tipo di paziente, della sua patologia di base e del quadro clinico-strumentale. Un simile impiego di questi test richiederebbe sia un'elevatissima sensibilità del test panfungino, sia un suo costo decisamente inferiore a quello dei test specie-specifici. Sfortunatamente la situazione reale è alquanto differente.

Il test per il BDG ha sensibilità non ottimali, oscillanti tra l'80 ed il 100\%. Ciò nonostante un eventuale falso negativo del BDG, se ottenuto in presenza di un quadro clinico fortemente suggestivo di infezione fungina in atto, potrebbe comunque non interrompere l'iter diagnostico verso un test specie-specifico.

Il maggiore impedimento ad un uso della ricerca del BDG come test di screening è invece il suo costo, poiché un test per il BDG costa 30-40 €/campione contro i 5-10 €/campione dei test per il MAN e GM, mentre le ricerche anticorpali anti-Candida ed anti-Aspergillus costano ancora meno. Pertanto un impiego del test per il BDG per lo screening di infezioni micotiche appare economicamente molto impegnativo e non sempre sostenibile.

Un'alternativa potrebbe essere quella di usare il test panfungino per il BDG come integrazione dei metodi specie-specifici. In particolare, se il test per il BDG dimostrerà di non essere affetto dagli stessi problemi dei test per Candida (falsi positivi per colonizzazioni da Candida in assenza di infezioni) e dei test per Aspergillus (falsi negativi in pazienti trattati con antifungini o in pazienti con 
elevati titoli anticorpali anti-Aspergillus, e falsi positivi in pazienti pediatrici o trattati con $\beta$-lattamici), questo metodo potrà anzi avere un ruolo dirimente nei casi di risposte dubbie dei test specie-specifici.

\section{CONCLUSIONI}

Nonostante il grande sforzo nel cercare un'alternativa alla diagnostica tradizionale di infezione fungina, l'attuale gold standard resta la positività colturale ed istologica. Ad ogni modo le nuove metodiche antigeniche/anticorpali disponibili appaiono come un valido ed utile supporto alla diagnosi di micosi invasiva. Inoltre, il loro uso ormai diffuso ha permesso di scoprire e chiarire aspetti molecolari, fisiologici ed epidemiologici delle micosi opportunistiche finora sconosciuti.

Molta aspettativa c'è anche nei riguardi della diagnostica molecolare delle infezioni fungine. Questo approccio finora ha prodotto solo sistemi non standardizzati, i quali molto spesso non varcano i confini dei laboratori in cui sono messi a punto. Recentemente però qualcosa sta cambiando, e circa 15 anni di ricerche cominciano a produrre alcuni metodi più standardizzati che ora necessiteranno di ampie validazioni cliniche.

Il futuro della diagnosi di infezione fungina invasiva si profila pertanto sempre più come un pannello integrato di sistemi diagnostici tradizionali e innovativi.

Un uso appropriato dei metodi disponibili e la loro corretta interpretazione ed applicazione alla clinica, si spera consentiranno presto una diagnosi più rapida ed affidabile delle infezioni micotiche invasive.

\section{BIBLIOGRAFIA}

1. Adam O, Auperin A, Wilquin F et al. Treatment with Piperacillin-Tazobactam and false-positive Aspergillus galactomannan antigen test results for patients with hematological malignancies. Clin Infect Dis 2004; 38: 917 - 20

2. Ahmad S, Khan Z, Mustafa AS, et al. Seminested PCR for diagnosis of candidemia: comparison with culture, antigen detection, and biochemical methods for species identification. J Clin Microbiol 2002; 40: $2483-9$

3. Allan EK, Jordanides NE, McLintock LA, et al. Poor performance of galactomannan and mannan sandwich enzyme-linked immunosorbent assays in the diagnosis of invasive fungal infection. $\mathrm{Br} J$ Haematol 2005; 128: 578 - 9

4. Ansorg R, van den Boom R, Rath PM. Detection of Aspergillus galactomannan antigen in foods and antibiotics. Mycoses 1997; 40: 353-7

5. Aubry A, Porcher R, Bottero J, et al. Occurrence and kinetics of false-positive Aspergillus galactomannan test results following treatment with $\beta$-lactam antibiotics in patients with hematological disorders. J Clin
Microbiol 2006; 44: 389 - 94

6. Bar W, Hecker H. Diagnosis of systemic Candida infections in patients of the intensive care unit. Significance of serum antigens and antibodies. Mycoses 2002; 45: 22-8

7. Bart-Delabesse E, Basile M, Al Jijakli A, et al. Detection of Aspergillus galactomannan antigenemia to determine biological and clinical implications of Beta-lactam treatments. $J$ Clin Microbiol 2005; 43: $5214-20$

8. Becker MJ, Lugtenburg EJ, Cornelissen JJ, et al. Galactomannan detection in computerized tomography-based broncho-alveolar lavage fluid and serum in haematological patients at risk for invasive pulmonary aspergillosis. Br J Haematol 2003; 121: 448 - 57

9. Berenguer J, Allende MC, Lee JW. Pathogenesis of pulmonary aspergillosis. Granulocytopenia versus cyclosporine and methylprednisolone-induced immuno-suppression. Am J Respir Crit Care Med 1995; 152: 1079 - 86

10. Bialek R, Moshous D, Casanova JL, et al. Aspergillus antigen and PCR assays in bone marrow transplanted children. Eur J Med Res 2002; 7: 177 - 80

11. Boutboul F, Alberti C, Leblanc T, et al. Invasive aspergillosis in allogeneic stem cell transplant recipients: increasing antigenemia is associated with progressive disease. Clin Infect Dis 2002; 34: 939-43

12. Bretagne S, Marmorat-Khuong A, Kuentz M, et al. Serum Aspergillus galactomannan antigen testing by sandwich ELISA: practical use in neutropenic patients. J Infect 1997; 35: 7 - 15

13. Caillot D, Casasnovas O, Bernard A, et al. Improved management of invasive pulmonary aspergillosis in neutropenic patients using early thoracic computed tomographic scan and surgery. J Clin Oncol 1997; 15: $139-47$

14. Dalle F, Charles PE, Blanc K, et al. Cryptococcus neoformans Galactoxylomannan Contains an Epitope(s) That Is Cross-Reactive with Aspergillus alactomannan. J Clin Microbiol 2005; 43: 2929 - 31

15. Dalle F, Lopez J, Caillot D, et al. False-positive results caused by cotton swabs in commercial Aspergillus antigen latex agglutination test. Eur $J$ Clin Microbiol Infect Dis 2002; 21: 130 - 2

16. De Repentigny L. Serodiagnosis of candidiasis, aspergillosis, and cryptococcosis. Clin Infect Dis 1992; 14(Suppl 1): S11-22

17. Digby J, Kalbfleisch J, Glenn A, et al. Serum glucan levels are not specific for presence of fungal infections in intensive care unit patients. Clin Diagn Lab Immunol 2003; 10: 882 - 5

18. Dupont B, Improvisi L, Provost F. Détection de galactomannane dans les aspergilloses invasives humaines et animales avec un test au latex. Bull Soc Fr Mycol Méd 1990; 19: 35 - 42

19. El Saleeby CM, Allison KJ, Knapp KM, et al. Discordant rise in galactomannan antigenemia in a patient with resolving aspergillosis, renal failure, and ongoing hemodialysis. J Clin Microbiol 2005; 43: 3560 - 3

20. Ellepola AN, Morrison CJ. Laboratory diagnosis of invasive candidiasis. J Microbiol 2005; 43: 65 - 84

21. Erjavec Z, Verweij PE. Recent progress in the diagnosis of fungal infections in the immunocompromised host. Drug Resist Updat 2002; 5: 3 - 10

22. Fortun J, Martin-Davila P, Alvarez ME, et al. The Ramon y Cajal Hospital's Liver Transplant Group. Aspergillus antigenemia sandwich-enzyme immunoassay test as a serodiagnostic method for invasive aspergillosis in liver transplant recipients. 
Transplantation 2001; 71: 145 - 9

23. Gangneux JP, Lavarde D, Bretagne et al. Transient Aspergillus antigenaemia: think of milk. Lancet 2002; 359: 1251

24. Gentry LO, Wilkinson ID, Lea AS, et al. Latex agglutination test for detection of Candida antigen in patients with disseminated disease. Eur J Clin Microbiol Infect Dis 1983; 2: 122-8

25. Giacchino M, Chiapello N, Bezzio S, et al. Aspergillus Galactomannan Enzyme-Linked Immunosorbent Assay Cross-Reactivity Caused by Invasive Geotrichum capitatum. J Clin Microbiol 2006; 44: 3432 - 4

26. Groll AH, Shah PM, Mentzel C, et al. Trends in the postmortem epidemiology of invasive fungal infections at a university hospital. J Infect 1996; 33: $23-32$

27. Hamaki T, Kami M, Kanda Y, et al. False positive results of Aspergillus enzyme-linked immunosorbent assay in a patient with chronic graft-versus-host disease after allogeneic bone marrow transplantation. Bone Marrow Transplant 2001; 28: 633 - 4

28. Hashiguchi K, Niki Y, Soejima R. Cyclophosphamide induces false-positive results in detection of Aspergillus antigen in urine. Chest 1994; 105: 975

29. Herbrecht R, Letscher-Bru V, Oprea C, et al. Aspergillus galactomannan detection in the diagnosis of invasive aspergillosis in cancer patients. J Clin Oncol 2002; 20: 1898 - 906

30. Herent P, Stynen D, Hernando F, Fruit J, et al. Retrospective evaluation of two latex agglutination tests for detection of circulating antigens during invasive candidosis. J Clin Microbiol 1992; 30: 2158 - 64

31. Hope WW, Walsh TJ, Denning DW. Laboratory diagnosis of invasive aspergillosis. Lancet Infect Dis 2005; 5: $609-22$

32. Hopwood V, Johnson EM, Cornish JM, et al. Use of the Pastorex Aspergillus antigen latex agglutination test for the diagnosis of invasive aspergillosis. J Clin Pathol 1995; 48: 210 - 3

33. Hurst SF, Reyes GH, McLaughlin DW, et al. Comparison of commercial latex agglutination and sandwich enzyme immunoassays with a competitive binding inhibition enzyme immunoassay for detection of antigenemia and antigenuria in a rabbit model of invasive aspergillosis. Clin Diagn Lab Immunol 2000; 7: $477-85$

34. Husain S, Kwak EJ, Obman A, et al. Prospective Assessment of Platelia ${ }^{\mathrm{TM}}$ Aspergillus Galactomannan Antigen for the Diagnosis of Invasive Aspergillosis in Lung Transplant Recipients. Am J Transplant 2004; 4: $796-802$

35. Iwasaki H, Misaki H, Nakamura T, et al. Surveillance of the serum Candida antigen titer for initiation of antifungal therapy after post-remission chemotherapy in patients with acute leukemia. Intl J Hematol 2000; 71: $266-72$

36. Jones JM. Kinetics of antibody responses to cell wall mannan and a major cytoplasmic antigen of Candida albicans in rabbits and humans. J Lab Clin Med 1980; 96: 845 - 60

37. Kami M, Kanda Y, Ogawa S, et al. Frequent false-positive results of Aspergillus latex agglutination test: transient Aspergillus antigenemia during neutropenia. Cancer 1999; 86: 274 - 81

38. Kami M, Ogawa S, Kanda Y, et al. Early diagnosis of central nervous system aspergillosis using polymerase chain reaction, latex agglutination test, and enzyme linked immunosorbent assay. Br J Haematol 1999; 106: $536-7$
39. Kappe R, Muller J. Rapid clearance of Candida albicans mannan antigens by liver and spleen in contrast to prolonged circulation of Cryptococcus neoformans antigens. J Clin Microbiol 1991; 29: 1665 - 9

40. Kappe R, Schulze-Berge A. New cause for false positive results with the Pastorex Aspergillus antigen latex agglutination test. J Clin Microbiol 1993; 31: $2489-90$

41. Kawazu M, Kanda Y, Nannya Y, et al. Prospective comparison of the diagnostic potential of real-time PCR, double-sandwich enzyme-linked immunosorbent assay for galactomannan, and a $(1 \rightarrow 3)-\beta$-D-glucan test in weekly screening for invasive aspergillosis in patients with hematological disorders. J Clin Microbiol 2004; 42: 2733 - 41

42. Kwak EJ, Husain S, Obman A, et al. Efficacy of galactomannan antigen in the Platelia Aspergillus enzyme immunoassay for diagnosis of invasive aspergillosis in liver transplant recipients. J Clin Microbiol 2004; 42: $435-8$

43. Latge JP. Aspergillus fumigatus and aspergillosis. Clin Microbiol Rev 1999; 12: 310 - 50

44. Letscher-Bru V, Cavalier A, Pernot-Marino E, et al. Recherche d'antigène galactomannane aspergillaire circulant par Platelia Aspergillus: antigénémies positivies en l'absence d'infection. J Mycol Méd 1998; 8: $112-3$

45. Machetti M, Di Domenico S, Pellizzari A, et al. Galactomannan and mannan antigens and anti-mannan antibodies detection for the diagnosis of invasive aspergillosis and candidiasis in liver transplant recipients. $16^{\text {th }}$ ECCMID, Nice, France, 2006

46. Machetti M, Feasi M, Mordini N, et al. Comparison of an enzyme immunoassay and a latex agglutination system for the diagnosis of invasive aspergillosis in bone marrow transplant recipients. Bone Marrow Transplant 1998; 21: 917 - 21

47. Machetti M, Furfaro E, Caviglia I, et al. Six-Years Use of Galactomannan Detection for the Diagnosis of Invasive Aspergillosis in Paediatric Patients. $46^{\text {th }}$ $I C A A C$, San Francisco, U.S.A., 2006

48. Machetti M, Furfaro E, Viscoli C. Galactomannan in Piperacillin-Tazobactam: How Much and to What Extent?. Antimicrob Agents Chemother 2005; 49: $3984-5$

49. Machetti M, Majabo MJ, Furfaro E, et al. Kinetics of galactomannan in surgical patients receiving perioperative piperacillin/tazobactam prophylaxis. J Antimicrobial Chemotherapy 2006; 58: 806 - 10

50. Maertens J, Theunissen K, Verbeken E, et al. Prospective clinical evaluation of lower cut-offs for galactomannan detection in adult neutropenic cancer patients and haematological stem cell transplant recipients. Br J Haematol 2004; 126: 852 - 60

51. Maertens J, Van Eldere J, Verhaegen J, et al. Use of circulating galactomannan screening for early diagnosis of invasive aspergillosis in allogeneic stem cell transplant recipients. J Infect Dis 2002; 186: 1297 - 306

52. Maertens J, Verhaegen J, Lagrou K, et al. Screening for circulating galactomannan as a noninvasive diagnostic tool for invasive aspergillosis in prolonged neutropenic patients and stem cell transplantation recipients: a prospective validation. Blood 2001; 97: 1604 - 10

53. Marino IR, Panarello G, Singh N. Efficacy of Aspergillus galactomannan-directed preemptive therapy for the prevention of invasive aspergillosis in organ transplant recipients. Transpl Infect Dis 2002; 4: 226 - 7

54. Marr KA, Balajee SA, McLaughlin L, et al. Detection of galactomannan antigenemia by enzyme immunoassay for the diagnosis of invasive aspergillosis: vari- 
ables that affect performance. J Infect Dis 2004; 190: $641-9$

55. Marr KA, Laverdiere M, Gugel A, et al. Antifungal therapy decreases sensitivity of the Aspergillus galactomannan enzyme immunoassay. Clin Infect Dis 2005; 40: 1762 - 9

56. Mattei D, Rapezzi D, Mordini N, et al. False-positive Aspergillus galactomannan enzyme-linked immunosorbent assay results in vivo during amoxicillin-clavulanic acid treatment. J Clin Microbiol 2004; 42: 5362 - 3

57. Mennink-Kersten MA, Donnelly JP, Verweij PE. Detection of circulating galactomannan for the diagnosis and management of invasive aspergillosis. Lancet Infect Dis 2004; 4: 349 - 57

58. Mennink-Kersten MA, Klont RR, Warris A, et al. Bifidobacterium lipoteichoic acid and false ELISA reactivity in Aspergillus antigen detection. Lancet 2004; 363: 325 - 7

59. Mennink-Kersten MA, Warris A, Verweij PE. 1,3- $\beta$-Dglucan in patients receiving intravenous amoxicillinclavulanic acid. $N$ Engl J Med 2006; 354: 2834 - 5

60. Mennink-Kersten MAS, Klont RR, Ruege-Brink D, Op den Camp H, Verweij PE. Amoxicillin-clavulanic acid (AMC) and piperacillin-tazobactam (PTZ) contain high molecular weight cross reacting Aspergillus antigen. $44^{\text {th }} I C A A C$, Washington, DC, U.S.A., 2004

61. Metan G, Durusu M, Uzun O. False positivity for Aspergillus antigenemia with amoxicillin-clavulonic acid. J Clin Microbiol 2005; 43: 2548 - 9

62. Meunier F, Viscoli C. Micosi. In: Trattato delle Malattie Infettive, Terragna A, Di Nola F Eds. UTET, 1992: 547-60

63. Misaki H, Iwasaki H, Ueda T. A comparison of the specificity and sensitivity of two Candida antigen assay systems for the diagnosis of deep candidiasis in patients with hematologic diseases. Med Sci Monit 2003; 9: 1-7

64. Mitsutake K, Miyazaki T, Tashiro T, et al. Enolase antigen, mannan antigen, Cand-Tec antigen, and $\beta$ glucan in patients with candidemia. J Clin Microbiol 1996; 38: 1918 - 21

65. Murashige N, Kami M, Kishi Y, et al. False-positive results of Aspergillus enzyme-linked immunosorbent assays for a patient with gastrointestinal graft-versushost disease taking a nutrient containing soybean protein. Clin Infect Dis 2005; 40: 333 - 4

66. Musher B, Fredricks D, Leisenring W, et al. Aspergillus galactomannan enzyme immunoassay and quantitative PCR for diagnosis of invasive aspergillosis with bronchoalveolar lavage. J Clin Microbiol 2004; 42: 5517 - 22

67. Na BK, Song CY. Use of monoclonal antibody in diagnosis of candidiasis caused by Candida albicans: detection of circulating aspartyl proteinase antigen. Clin Diagn Lab Immunol 1999; 6: 924 - 9

68. Obayashi T, Yoshida M, Mori T, et al. Plasma $(1 \rightarrow 3)$ $\beta$-D-glucan measurement in diagnosis of invasive deep mycosis and fungal febrile episodes. Lancet 1995; 345: 17 - 20

69. Ormala T, Korppi M, Katila ML, et al. Prospective evaluation of candida antigen and antibody assays for detection of candida infections in children with malignant disease. Acta Paediatr 1995; 84: 183 - 7

70. Ostrosky-Zeichner L, Alexander BD, Kett DH, et al. Multicenter clinical evaluation of the $(1 \rightarrow 3)-\beta$-D-glucan assay as an aid to diagnosis of fungal infections in humans. Clin Infect Dis 2005; 41: 654 - 9

71. Pallavicini F, Izzi I, Pennisi MA, et al. Evaluation of the utility of serological tests in the diagnosis of candidemia. Minerva Anestesiol 1999; 65: 637 - 9
72. Penack O, Schwartz S, Thiel E, et al. Lack of evidence that false-positive Aspergillus galactomannan antigen test results are due to treatment with piperacillintazobactam. Clin Infect Dis 2004; 39: 1401 - 2

73. Pickering JW, Sant HW, Bowles CA, et al. Evaluation of a $(1 \rightarrow 3)-\beta-D-G l u c a n$ Assay for Diagnosis of Invasive Fungal Infections. J Clin Microbiol 2005; 43: $5957-62$

74. Poulain D, Robert R, Mesnard F, et al. Clearances of Candida albicans-derived alpha- and beta-linked mannose residues in sera from patients with candidiasis. Eur J Clin Microbiol Infect Dis 1997; 16: 16 - 20

75. Prella M, Bille J, Pugnale M, et al. Early diagnosis of invasive candidiasis with mannan antigenemia and antimannan antibodies. Diagn Microbiol Infect Dis 2005; 51: 95 - 101

76. Reiss E, Lehmann PF. Galactomannan antigenemia in invasive aspergillosis. Infect Immun 1979; 25: 357 - 65.

77. Reiss E, Morrison CJ. Nonculture methods for diagnosis of disseminated candidiasis. Clin Microbiol Rev 1993; 6: $311-23$

78. Rimek D, Singh J, Kappe R. Cross-reactivity of the platella candida antigen detection enzyme immunoassay with fungal antigen extracts. J Clin Microbiol 2003; 41: $3395-8$

79. Rogers TR, Haynes KA, Barnes RA. Value of antigen detection in predicting invasive pulmonary aspergillosis. Lancet 1990; 336: 1210 - 3

80. Rohrlich P, Sarfati J, Mariani P, et al. Prospective sandwich enzyme-linked immunosorbent assay for serum galactomannan: early predictive value and clinical use in invasive aspergillosis. Pediatr Infect Dis $\mathbf{J}$ 1996; 15: 232 - 7

81. Salonen J, Lehtonen OP, Terasjarvi MR, et al. Aspergillus antigen in serum, urine and bronchoalveolar lavage specimens of neutropenic patients in relation to clinical outcome. Scand J Infect Dis 2000; 32: $485-90$

82. Sendid B, Jouault T, Coudriau R, et al. Increased sensitivity of mannanemia detection tests by joint detection of $\alpha$ - and $\beta$-linked oligomannosides during experimental and human systemic candidiasis. J Clin Microbiol 2004; 42: 164 - 71

83. Sendid B, Poirot JL, Tabouret M, et al. Combined detection of mannanaemia and antimannan antibodies as a strategy for the diagnosis of systemic infection caused by pathogenic Candida species. J Med Microbiol 2002; 51: 433 - 42

84. Sendid B, Tabouret M, Poirot JL, et al. New enzyme immunoassays for sensitive detection of circulating Candida albicans mannan and antimannan antibodies: Useful combined test for diagnosis of systemic candidiasis. J Clin Microbiol 1999; 37: 1510 - 7

85. Severens JL, Donnelly JP, Meis JFGM, et al. Two strategies for managing invasive aspergillosis: a decision analysis. Clin Infect Dis 1997; 25: 1148 - 54

86. Siemann M, Koch-Dorfler M. The Platelia Aspergillus ELISA in diagnosis of invasive pulmonary aspergilosis (IPA). Mycoses 2001; 44: 266 - 72

87. Siemann M, Koch-Dorfler M, Gaude M. False-positive results in premature infants with the Platelia Aspergillus sandwich enzyme-linked immunosorbent assay. Mycoses 1998; 41: 373 - 7

88. Singh N, Obman A, Husain S, et al. Reactivity of platelia Aspergillus galactomannan antigen with piperacillin-tazobactam: clinical implications based on achievable concentrations in serum. Antimicrob Agents Chemother 2004; 48: 1989 - 92

89. Stevens DA. Diagnosis of fungal infections: current status. J Antimicrob Chemother 2002; 49: 11 - 9 
90. Stynen D, Goris A, Sarfati J, et al. A new sensitive sandwich enzyme-linked immunosorbent assay to detect galactofuran in patients with invasive aspergillosis. J Clin Microbiol 1995; 33: 497 - 500

91. Stynen D, Sarfati J, Goris A, et al. Rat monoclonal antibodies against Aspergillus galactomannan. Infect Immun 1992; 60: 2237 - 45

92. Sulahian A, Boutboul F, Ribaud P, et al. Value of antigen detection using an enzyme immunoassay in the diagnosis and prediction of invasive aspergillosis in two adult and pediatric hematology units during a 4year prospective study. Cancer 2001; 91: 311 - 8

93. Sulahian A, Touratier S, Leblanc T, et al. False Positive Aspergillus Antigenemia Related To Concomitant Administration Of Tazocillin. 43 ICAAC, Chicago, USA, 2003

94. Sulahian A, Touratier S, Ribaud P. False positive test for Aspergillus antigenemia related to concomitant administration of piperacillin and tazobactam. $N$ Engl $J$ Med 2003; 349: 2366 - 7

95. Swanink CM, Meis JF, Rijs AJ, et al. Specificity of a sandwich enzyme-linked immunosorbent assay for detecting Aspergillus galactomannan. J Clin Microbiol 1997; 35: 257 - 60

96. Tanriover MD, Metan G, Altun B, et al. False positivity for Aspergillus antigenemia related to the administration of piperacillin/tazobactam. Eur J Intern Med 2005; 16: 489 - 91

97. Verduyn-Lunel FM, Voss A, Kuijper EJ, et al. Detection of the Candida Antigen Mannan in Cerebrospinal Fluid Specimens from Patients Suspected of Having Candida Meningitis. J Clin Microbiol 2004; 42: 867 - 70

98. Verweij PE, Brinkman K, Kremer HPH, et al. Aspergillus meningitis: diagnosis by non-culturebased microbiological methods and management. J Clin Microbiol 1999; 37: 1186 - 9

99. Verweij PE, Stynen D, Rijs AJMM, et al. Sandwich enzyme-linked immunosorbent assay compared with Pastorex latex agglutination test for diagnos- ing invasive aspergillosis in immunocompromised patients. J Clin Microbiol 1995; 33: 1912 - 4

100. Viscoli C, Castagnola E, Zotti M. Micosi opportunistiche, p. 1 - 15. In Le infezioni fungine di interesse medico: quadri clinici, diagnosi, terapia. Il Pensiero Scientifico Editore. 2000

101. Viscoli C, Machetti M, Cappellano P, et al. FalsePositive Platelia Aspergillus (PA) Test in Patients (pts) Receiving Piperacillin-Tazobactam (P/T). $43^{\text {rd }}$ ICAAC, Chicago, USA, 2003

102. Viscoli C, Machetti M, Cappellano P, et al. False-positive galactomannan Platelia Aspergillus test results for patients receiving piperacillin-tazobactam. Clin Infect Dis 2004; 38: 913 - 5

103. Viscoli C, Machetti M, Gazzola P, et al. Aspergillus galactomannan antigen in the cerebrospinal fluid of bone marrow transplant recipients with probable cerebral aspergillosis. J Clin Microbiol 2002; 40: $1496-9$

104. Walsh TJ, Pizzo P A. Laboratory diagnosis of candidiasis. In: Candidiasis, pathogenesis, diagnosis and treatment, Bodey GP Ed, Second Edition. Raven Press, 1993; 109 - 35

105. Walsh TJ, Shoham S, Petraitiene R, et al. Detection of galactomannan antigenemia in patients receiving piperacillin-tazobactam and correlations between in vitro, in vivo, and clinical properties of the drug-antigen interaction. J Clin Microbiol 2004; 42: $4744-8$

106. Yeo SF, Wong B. Current status of nonculture methods for diagnosis of invasive fungal infections. Clin Microbiol Rev 2002; 15: 465 - 84

107. Yera H, Sendid B, Francois N, et al. Contribution of serological tests and blood culture to the early diagnosis of systemic candidiasis. Eur J Clin Microbiol Infect Dis 2001; 20: 864 - 70

108. Yoshida K, Niki Y, Mitekura H, et al. A discrepancy in the values of serum $(1 \rightarrow 3)-\beta$-D-glucan measured by two kits using different methods. Nippon Ishinkin Gakkai Zasshi 2001; 42: 237 - 42 